\section{SUSSOL - Using AI for the selection and substitution of greener solvent}

Solvents can be toxic, volatile, highly flammable, non-biodegradable and nonrenewable. Searching for a suitable green alternative for a nonbenign solvent can be Herwig Herwig De Smet and Dr Jeroe University of Applied Sciences and Arts, in close collaboration with their industrial partners, have developed a user-

friendly software tool based on an Al algorithm, called SUSSOL (Sustainable Solvent Selection and Substitution more efficient objective and purposeful selection of the best-performing and most sustainable solvents for specific industrial applications.

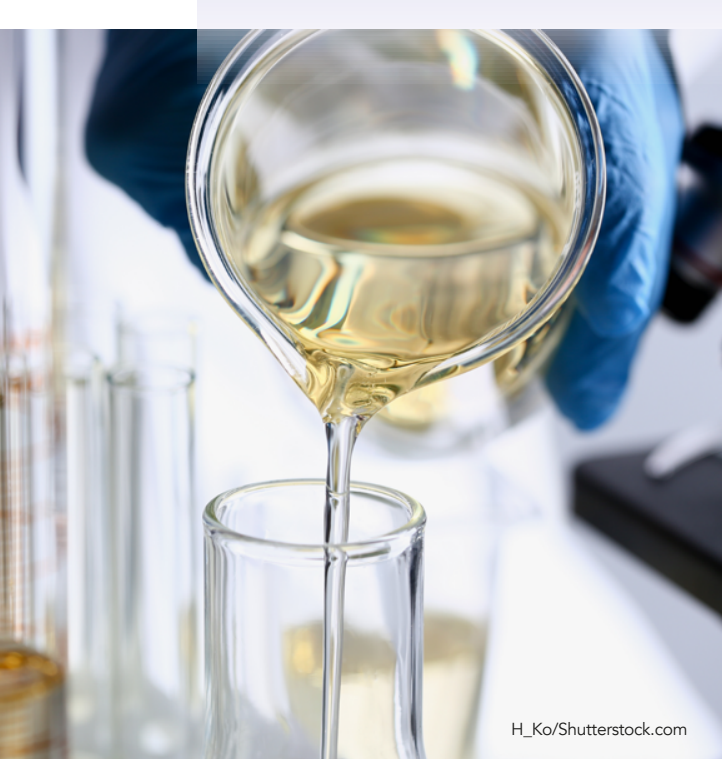

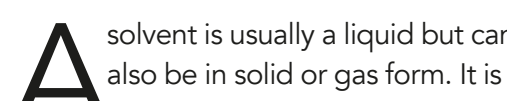
European regulation has been adopted to improve the protection of both used to dissolve, suspend and change to either the solvent or the other materials. Varied and versatile, solvents are used in the manufacture of a wide range of everyday products, including paint, pharmaceuticals, pesticides, cleaners, and inks. Numerous different solvents are used to meet specific requirements in order to make products with optimal performance attributes, such as inks that don't smudge, cleaning agents that remove grease and dirt effectively, and spray paints that dry quickly, last a long time, and don't clog the spray nozzle. Traditional solvents can be toxic, volatile, highly flammable, non-biodegradable and non-renewable. Searching for a suitable be difficult. Finding a green alternative for a nonbenign solvent can be even harder. Hannes Sels, Henvig De Smet and Dr Jeroen Geuens, from the Centre of Expertise on Sustainable Chemistry at Karel de Grote University of Applied Sciences and Arts in Antwerp, have developed a user-friendly software tool based on an Artificial Intelligence (A) algorithm, called SUSSOL (Sustainable Solvents Selection and Substitution Software), that enables a more efficient, objective and purposeful selection of the best performing and most sustainable CHEMICAL LEGISLATION CHEMICAL LEGISLATION
The Registration, Evaluation, Authorisation and Restriction of Chemicals (REACH)

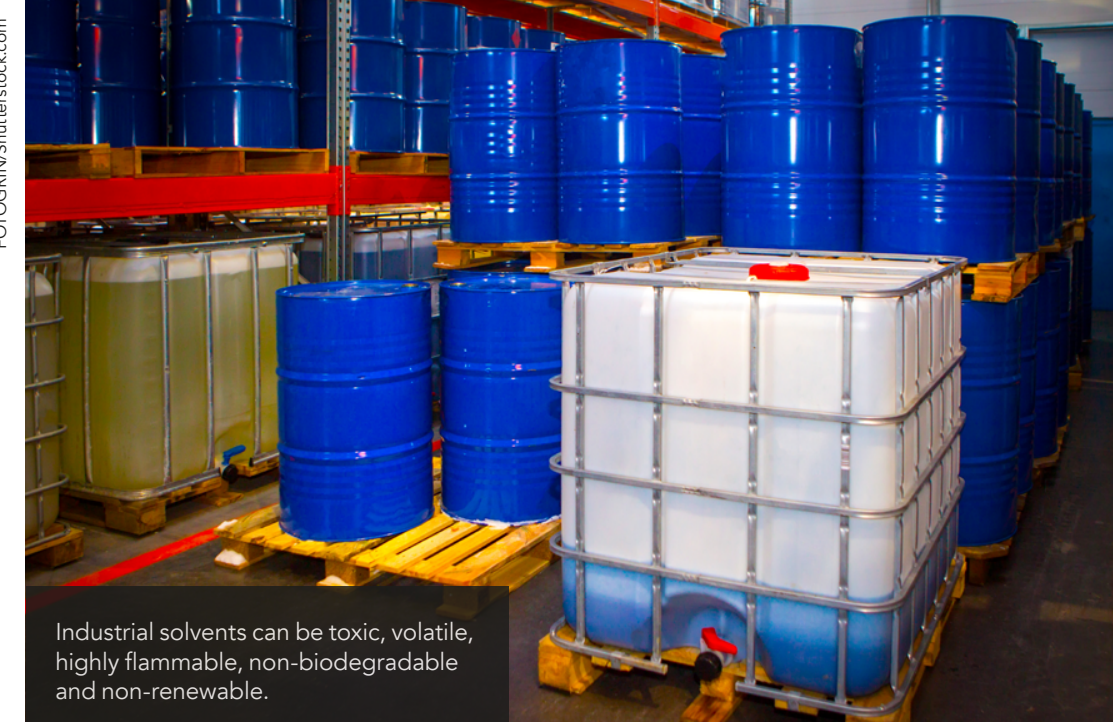

guide would allow users, unfamiliar with the field of solvent selection, to search for greener solvents and thus creating more s and processes.

A variety of sustainable solvent alternatives e available but finding the most suitable or a particular manufacturing process is on time consuming, labour intensive, SME's (small and medium-sized enterprises) and small formulating companies where chemistry is not the core business may not have the means or knowledge to search for new and more sustainable solvents. A software-aided tool could be of great benefit to those companies that are unaware of the relevant physical solvent properties haracterising their products and processes. SUSSOL

Sels, De Smet and Dr Geuens have used Al to develop a user-friendly software too Amed SUSSOL (Sustainable Solvents which enats a more efficotware), and purposeful selection of solvents. SUSSOL provides support for businesses in their search for sustainable and viable alternatives for the nonbenign solvents they currently use in their products and processes. By providing a flexible tool that companies can use according to their own needs, SUSSOL is bridging the gap between academic research and applicability in industry.

The researchers explain how they consider a solvent selection/substitution tool to be an interactive data-centred atalogue, comprising both conventonal and neoteric solvents, that directs 政 alternative. The process is effortless and insparent and offers the user flexibil in that they can use their own dataset, specific or confidential data. Solvent

A data-driven, automated solvent selection/substitution guide would allow users, unfamiliar with the field of solvent selection, to search for greener solvents.

producers and distributors of green and neoteric solvents can also use the tool as a benchmark for the promotion of their solvents as an alternative for conventional nonbenign solvents.

This novel methodology for solvent selection and solvent substitutio

groups, of similar solvents. These clusters are based on the physical properties of the solvents and are presented in user-friendly visualisations. SUSSOL uses Kohonen's Self-organizing Map to carry rant distec ce between two clustes fornt distance between two clusters forming the form of Machine Learning algorithms, user friendly, robust and does not require

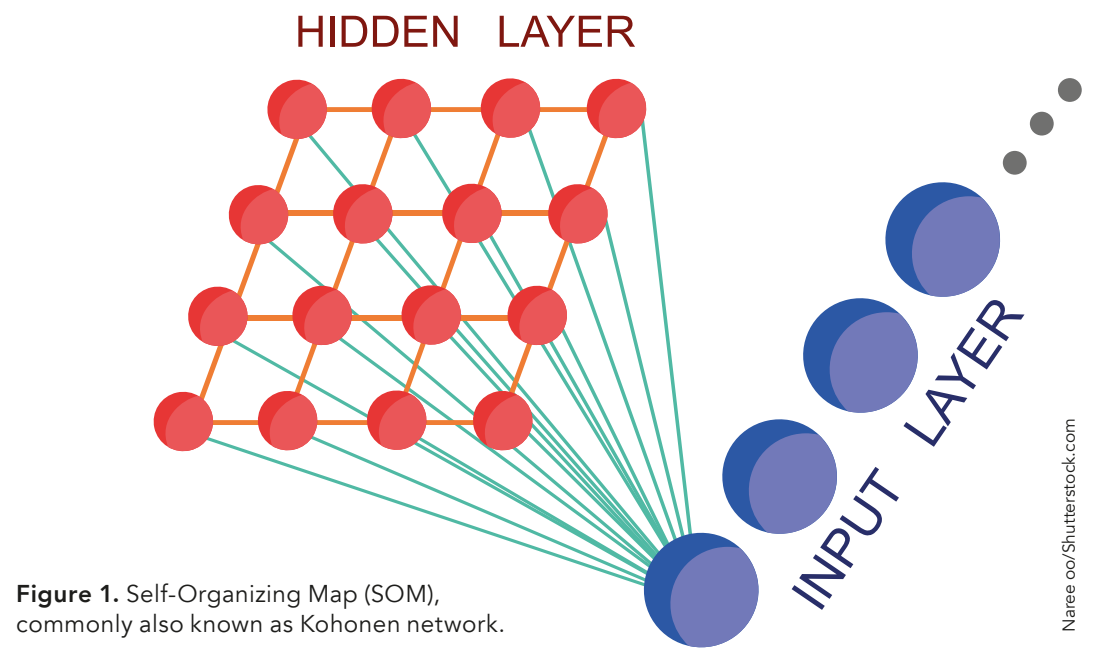




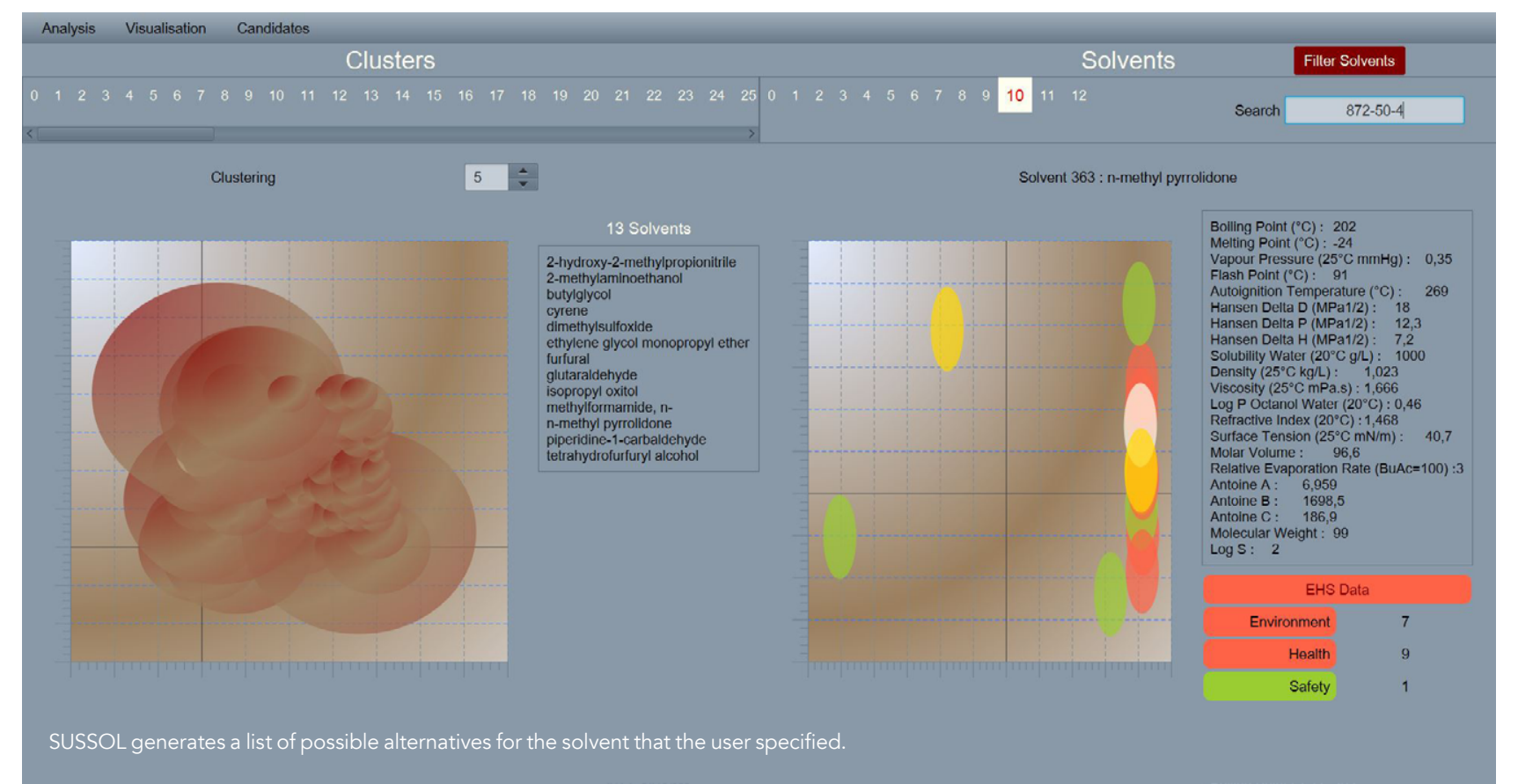

specific chemical knowledge. The user can specily the solvent they want to replace. SUSSOL generates a candidate list of similar solvents. The user can then select the most sustainable candidates and laboratory testing can be initiated.

Multidimensional Scaling is employed to visualise the clustering process. The user then selects a cluster and explores the cluster contents. Each solvent is colour coded, using the CHEM 21 methodology (CHEM 21 is a European public-private partnership which promotes sustainable biological and chemical methodologies). SUSSOL helps the user explore the solven space and generates and evaluates a list that the user specified The alvent that the userspected. The atsmatives health, and environment) scores.

\section{SOFTWARE VALIDATION}

The research team used case studies to demonstrate their SUSSOL approach and establish how it can help in the

greener solvents. To validate both the the clustering apal Scaling plot and organising Map algorithm, they sela number of case studies to display a variety of industrial applications.

The researchers demonstrate the use of SUSSOL in finding a greener alternative for cellulose thinners as a cleaning agen for ink in screen printing, for toluene in a contact adhesive and for selecting medium for an active pharmaceutical

similarities being observed overall. SUSSOL software makes intuitive sense. In the majority of cases, the software confirms the findings in literature, confirming that SUSSOL provides a sound platform for the selection of the most sustainable solvent candidate.

\section{COLLABORATION WITH} INDUSTRIAL PARTNERS SUSSOL was developed in close collaboration with the researchers industrial partners. This consortium covered the whole value chain

SUSSOL helps the user explore the solvent space and generates and evaluates a list of possible alternatives. ingredient synthesis. For each case, distributors, and end-users. After shortlist of possib solvents and display three years of intensive development the normalised values of a selection testing and statistical validation, the of physicochemical properties of each SUSSOL software tool was ready for solvents. In all applications, lab tests use. The initial results were promising: showed promising results with good SUSSOL has already been utilised to successfully determ alternative solvents for toluene in a contact adhesive, industrial cleaners and a chromatographic application. The collaborating companies, among others Soudal, eco-point, Caldic, Janssen Pharmaceuticals and Boss Paints, have identified sustainable solvents (sometimes as part of mixture) that meet their specific requests, either for substitution in

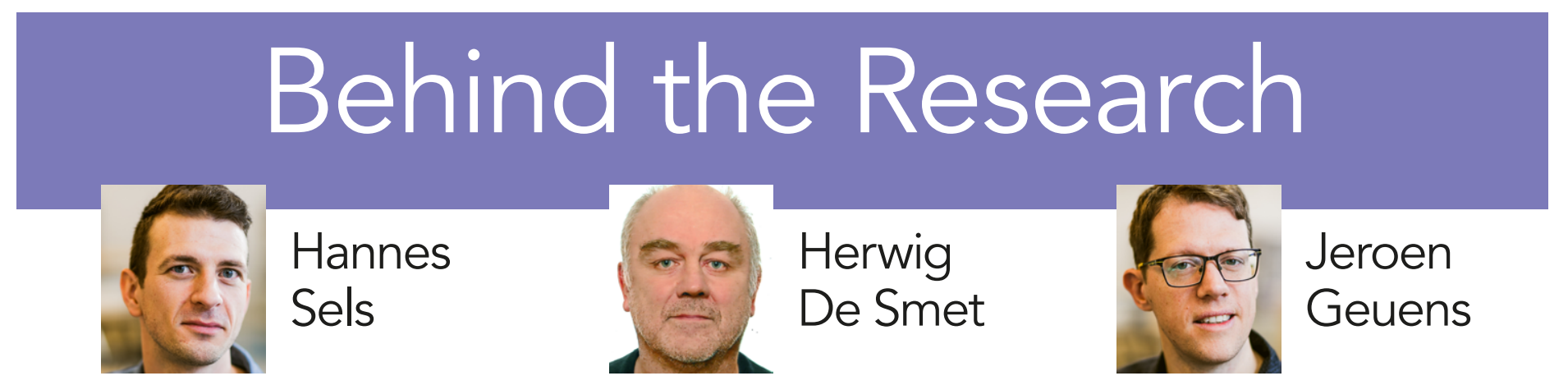

E: hannes.sels@kdg.be T: +3235022220 W: httpp://www.kdg.be/sustainable-chemistry/SUSSOL W: https://www.kdg be/onderzoek/expertisecentra/durzame-chemie

Research Objectives

The researchers describe a new methodology for solvent selection and substitution by applying Artificial Intelligence software to a database of solvents.

\section{Detail}

Address

Karel de Grote University of Applied Sciences and Arts Campus Hoboken
Salesianenlaan 90 2660 Antwerpen $\begin{array}{ll}\text { Bio } & \text { Sniversity of Applied } \\ \text { Biences and Arts } & \end{array}$ Chemistry works on employing Al models for optimist Chemistry works on employing Al models for optimising main goal is to make this technology available for SME's and non-research-intensive companies.

Funding

This research was supported by the Karel de Grote University of Applied Sciences and Arts (KdG) through funding by the Flemish government specifically allocated to Collaborators

Agfa, Boss paints, Caldic, Chemstream, eco-point, Janssen pharmaceutical companies, mcu coatings, Proviron,

"Surprisingly, SUSSOL can give you suggestions that you would not expect based on common knowledge and Jacco Quist - Product Manager Eco-Point

"A key feature of SUSSOL is that non-experts can readily get a good idea of likely safer solvents, just from the defaul solut few other general characteristics. Yet those who wish go deeper can refine the clustering according to their specific require

Prof Steven Abbot - Steven Abbott TCNF Ltd $=\mathrm{OH}_{4}+\mathrm{O}_{2} \Rightarrow \mathrm{Cl}$

\section{References}

Sels, H., De Smet, H., Geuens, J. (2020). SUSSOL - Using Artificial Intelligence for Greener Solvent Selection and Substitution. Molecules, 25(13), 3037. Avallable at:

https://doi.org/10.3390/molecules 25133037 Neural. Netw, 37, 52-65. Available at https://doi.org/10.1016/j.neunet.2012.09.018 Prat, D., Wells, A., Hayler, J., Sneddon, H., McElroy, C.R., Abou-Shehada, S., Dunn, P.J. (2015). CHEM21 selection guide of classical-and less classical-solvents. Green Chem., 18, 288-296.

\section{Personal Response}

What initially motivated you to develop SUSSOL?

II As a research group focusing on extraction of natural compounds, we used a lot of solvents such as hexane and We wanted to go greener. At the same time, we saw some companies in our network struggling with solventrelated issues. We realised that often there are greener alternative if you are not a solvent expert Together with colleagues from the Applied Informatics research group at $\mathrm{KdG}$ and a consortium of industrial partners,

we started the SUSSOL project.

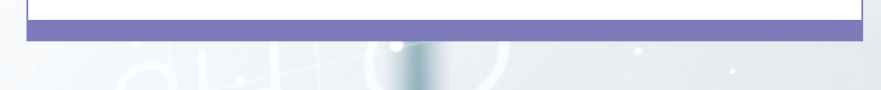

$2+12$

( 